\title{
Electric field mapping in CdSeTe solar cell using 4D-STEM
}

Jinglong Guo ${ }^{1}$, Ramesh Pandey ${ }^{2}$, Amit Munshi $^{2}$, Walajabad Sampath ${ }^{2}$ and Robert Klie ${ }^{3}$

${ }^{1}$ University of Illinois at Chicago, Cupertino, California, United States, ${ }^{2}$ Colorado State University, United States, ${ }^{3}$ University of Illinois at Chicago, Chicago, Illinois, United States

CdTe is the most promising material in solar cell applications due to its ideal band gap (1.5eV) and high absorption coefficient. However, the CdTe-based solar cells record laboratory efficiencies are still below the theoretical efficiency limit.[1] Research has shown that incorporating selenium into CdTe absorber significantly increases the minority carrier lifetime of device absorber, which is beneficial to solar cell open circuit voltage (Voc) and overall cell performance.[2], [3] In CdTe-based solar cells, carrier concentration in p-type absorber also plays an important role on open circuit voltage and conversion efficiency. Metzger et al. [4] have demonstrated in-situ arsenic doping in CdSeTe solar cells increases carrier concentration to $1016 \mathrm{~cm}-$ 3 , which leads to conversion efficiency of $20.8 \%$. With the increase in carrier concentration in the p-type absorber, it is necessary to increase carrier concentration in n-type buffer layer. In order to avoid interfacial recombination in front interface, with p-type concentration of $1016 \mathrm{~cm}-3$ in absorber, n-type carrier concentration in front buffer layer needs to be $1017 \mathrm{~cm}-3$. Gallium is believed to be a promising dopant in magnesium zinc oxide (MZO) buffer layer.[5] With such complicated multilayer doped structure, it is necessary to detect PN junction electric field distribution as results of gallium doping in MZO buffer layer.

This contribution will utilize 4D- scanning transmission electron microscopy (4D-STEM) to examine the atomic electric field as well as p/n-junction electric field distribution in CdSeTe-GMZO solar cell absorber. The 4D-STEM data is acquired by Gatan Continuum spectrometer on aberration-corrected electron microscope JEOL-JEM ARM200CF in UIC. Atomic ADF images and atomic electric field distribution are extracted from 4D-STEM data. Large scale p/n-junction electric field distribution is extracted, showing an electric field strength decay in absorber.

Low-magnification low angle annular dark field (LAADF) STEM image of CdSeTe-GMZO solar cell crosssection is shown in Figure 1. The $\mathrm{SnO} 2 \mathrm{TCO}$ and GaMgZnO layers are n-doped front contact. CdSeTe is ptype doped with $\mathrm{Cu}$. The 4D STEM measurement is applied to the area near front contact highlighted by the red square. The atomic ADF image is shown in Figure 2 (a). Figure 2 (b) shows CBED displacement mapping of the same area in Figure 2(a). The displacement vector can be decomposed into two direction: Horizontally the displacement vectors are convergent to each atom position, which is the result of atomic periodic electric field. Vertically the displacement is predominately pointing toward front contact, which is caused by the PNjunction electric field. The electric distribution within absorber is also examined using 4D-STEM, suggesting the $\mathrm{p} / \mathrm{n}$-junction electric field strength decays in the direction away from front contact.

In this contribution we demonstrate the direct detection of electric field strength distribution in CdSeTe solar cell using 4D-STEM. The 4D STEM technique can be used not only in GMZO characterization, but also other semiconductor device. This method provides a new way to probe electric field distribution in the semiconductor specimen at atomic scale, which is important for understand doping effects in semiconductor devices.[6] 


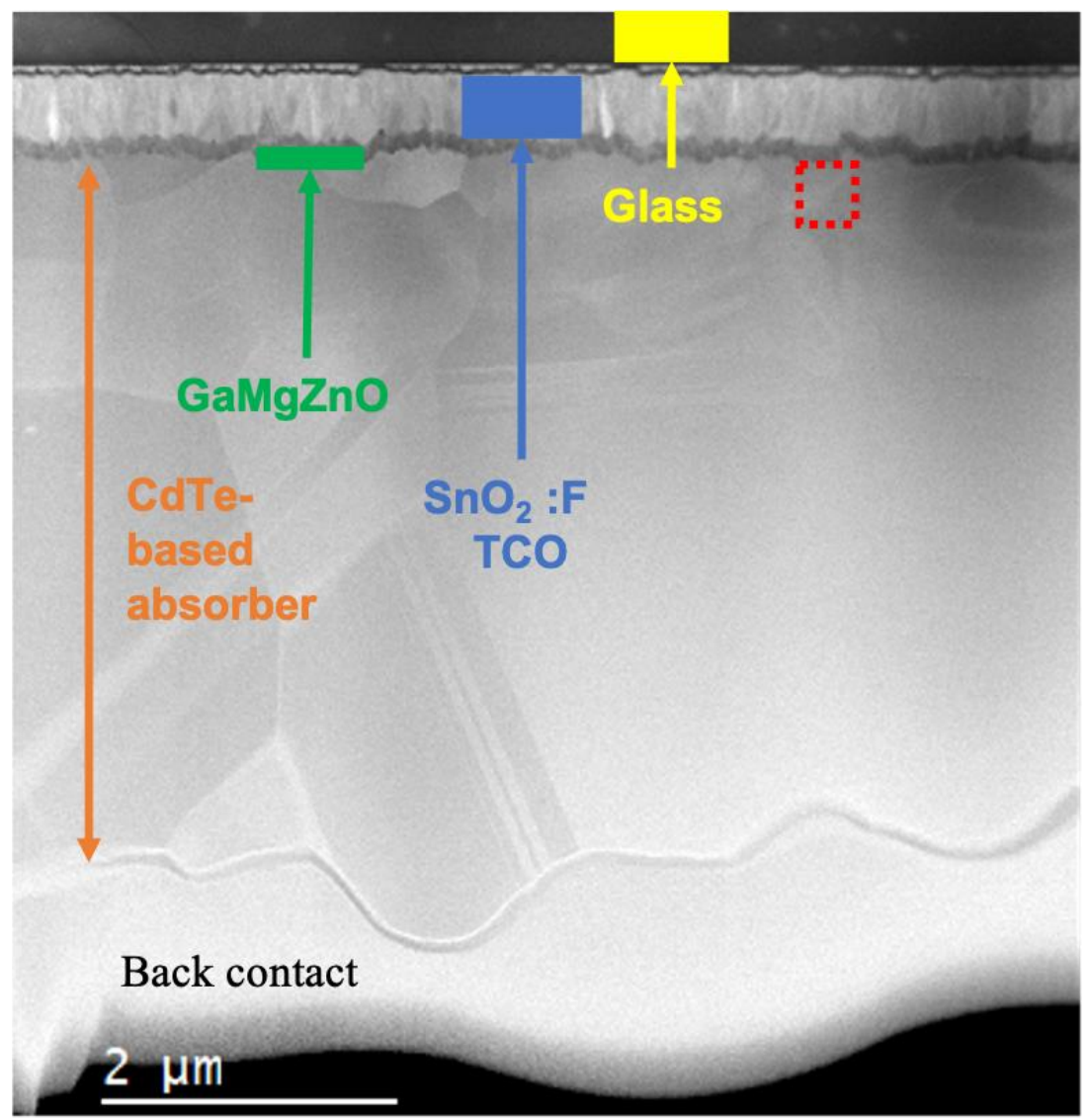

Figure 1. LAADF image of CdSeTe-GMZO solar cell.

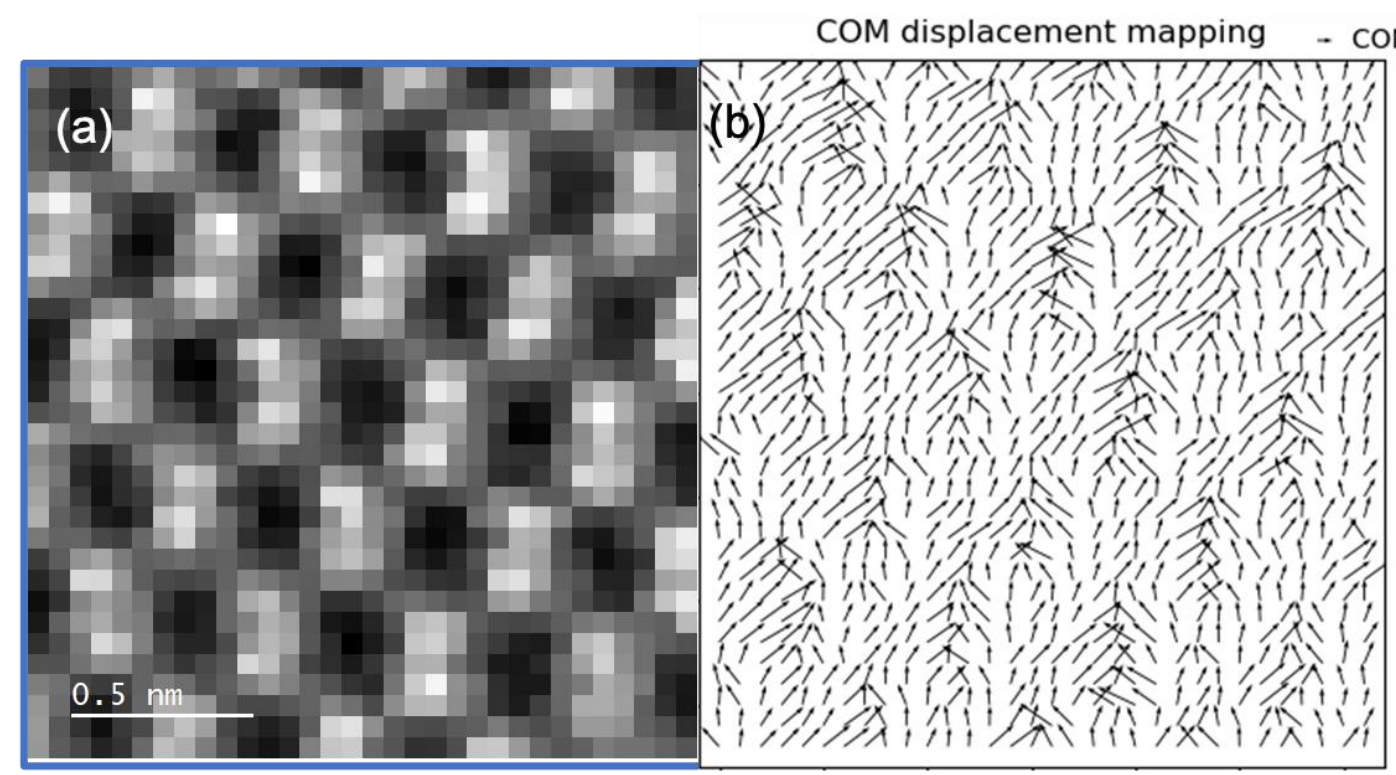

Figure 2. ADF image of region near front interface and corresponding CBED displacement mapping. 


\section{References}

[1] W. Shockley and H. J. Queisser, "Detailed balance limit of efficiency of p-n junction solar cells," J. Appl. Phys., vol. 32, no. 3, pp. 510-519, 1961, doi: 10.1063/1.1736034.

[2] T. A. M. Fiducia et al., "Understanding the role of selenium in defect passivation for highly efficient selenium-alloyed cadmium telluride solar cells," Nat. Energy, vol. 4, no. 6, pp. 504-511, 2019, doi: 10.1038/s41560-019-0389-z.

[3] J. Guo et al., "Effect of selenium and chlorine co-passivation in polycrystalline CdSeTe devices," Appl. Phys. Lett., 2019, doi: 10.1063/1.5123169.

[4] W. K. Metzger et al., "Exceeding 20\% efficiency with in situ group V doping in polycrystalline CdTe solar cells," Nat. Energy, pp. 37-39, 2019, doi: 10.1038/s41560-019-0446-7.

[5] R. Pandey, T. Shimpi, A. Munshi, and J. R. Sites, "Impact of Carrier Concentration and Carrier Lifetime on MgZnO/CdSeTe/CdTe Solar Cells," IEEE J. Photovoltaics, vol. 10, no. 6, pp. 1918-1925, Nov. 2020, doi: 10.1109/JPHOTOV.2020.3017741.

[6] This work was support by a grant from the Department of Energy, Solar Energy and Technology Office (SETO) witih grant number DE-EE0008557. 